\title{
Distribución termal intraurbana en las ciudades de Santiago y Valparaíso. Análisis comparativo de sus factores explicativos ${ }^{1}$
}

\author{
Pamela Smith² pamelasmit@gmail.com; Ximena Andrade ximena.andrade.c@gmail.com
}

\begin{abstract}
RESUMEN
Este trabajo se articula dentro de la temática del clima urbano, específicamente de la variabilidad del campo térmico a escala del dosel. Se analizan datos de temperatura atmosférica, en las ciudades de Santiago y Valparaíso, localizadas a una latitud similar, pero en el interior y en la costa respectivamente, durante el verano de los años 2009 y 2011. La multicausalidad del comportamiento de las temperaturas y su distribución espacial, se analizan considerando componentes naturales del paisaje, así como aquellos que guardan relación con el grado de perturbación urbana, en diferentes áreas, al interior de las ciudades. Se hace uso de técnicas asociadas al análisis espacial, estadística inferencial y en menor medida a la teledetección. Los resultados muestran en Santiago, la presencia de una isla de calor, que en la noche se manifiesta con mayor intensidad. Presenta un gradiente típico, que encuentra su máximo en las áreas centrales, lo que se corresponde en gran medida, con resultados obtenidos por investigaciones precedentes. El caso de Valparaíso es bastante similar durante la tarde y noche. El calor se concentra en el centro o Plan, decreciendo la intensidad de las temperaturas hacia la periferia. En ambos casos, la presencia y estado de la vegetación, así como, la proporción de superficies impermeabilizadas y construidas, son determinantes en la disminución e incremento de los registros respectivamente, por lo que se sugiere, que estas variables, sean consideradas como componentes estratégicos de la gestión y planificación ambiental de las ciudades.
\end{abstract}

Palabras clave: Clima Urbano, Temperatura Atmosférica, Regresión Múltiple, Santiago, Valparaíso.

\section{Intra urban thermal distributions in the cities of Santiago and Valparaíso. Comparative analysis of their explicative factors}

\begin{abstract}
This work fits into the theme of urban climate, specifically the variability of canopy-scale thermal field.. Data of atmospheric temperature are analyzed in the cities of Santiago and Valparaiso, located at similar latitude, but in the interior and on the coast, respectively, during the summer of 2009 and 2011. The multiple causes of temperature behavior and their spatial distribution are analyzed considering natural landscape components, as well as those related to the degree of urban disturbance in different areas within both cities. It makes use of techniques associated with the use of spatial analysis, statistical inference and to a lesser extent, to remote sensing. In Santiago, the results show the presence of a heat island, which manifests itself with greater intensity at night. It presents a typical gradient, which is a peak in the central areas of the city, which corresponds, largely, with results obtained by previous research. The case of Valparaiso, is fairly similar during the afternoon and evening. Heat is concentrated in the center or the city plan, decreasing the intensity of the temperatures toward the periphery. In both cases, the presence and state of the vegetation, as well as the proportion of waterproofed and built surfaces are crucial to the decrease and increase of the records respectively, so it is suggested that these variables are considered as strategic components of environmental management and planning of cities
\end{abstract}

Keywords: Urban Climate, Atmospheric Temperature, Multiple Regression, Santiago, Valparaíso.

Recibido el 21de octubre de 2013, aceptado el 20 de diciembre de 2013.

FONDECYT N 1100657 y 1130305.

Departamento de Geografía, Universidad de Chile. Portugal 084, Santiago, R.M. Chile. 


\section{INTRODUCCIÓN}

En el proceso de urbanización en Chile, se evidencian ciertas causas y tendencias asociadas al creciente y sostenido aumento de la población que ha migrado desde zonas rurales a zonas urbanas desde la década de 1940 en adelante (INTITUTO NACIONAL DE ESTADÍSTICAS, INE 2008), y a las modalidades de regulación dadas por los instrumentos de planificación, que han respondido muy ceñidamente a pautas económicas globales y locales referidas al proceso de globalización. La expansión de la superficie construida, ha tenido fuertes impactos en el territorio, y actualmente se reconoce que constituye un problema ambiental, cuando se desarrolla, desconociendo los mecanismos bajo los cuales se relacionan, funcionan $\mathrm{y}$ autorregulan los componentes naturales del paisaje, que serán alterados por dicho proceso. Ello, porque se perturba y limita la capacidad de los mismos para otorgar servicios ambientales, condicionando la calidad de vida de las personas que se benefician de ellos, y porque los esfuerzos abocados a conservarlos e incrementarlos, pueden llegar a ser ineficientes e incluso totalmente ineficaces.

La climatología urbana, es una de las disciplinas que ha intentado comprender los vínculos entre el clima y los diferentes componentes naturales y artificiales que configuran el medio ambiente urbano. Actualmente, existe una amplia gama de teorías y reconocimiento de patrones, asociados a la variabilidad climática que son comunes y propios de las ciudades. Cuestiones como la escala de análisis y el reconocimiento de capas de influencia, son consideradas determinantes en los vínculos que se establecen y resultados que se discuten, y desde la publicación del primer Informe sobre Cambio Climático (IPCC) en 1990, la mitigación de impactos y adaptación a cambios, se ha considerado un objetivo primordial a incorporar en materia de planificación urbana.
El clima puede ser definido, como el conjunto fluctuante de condiciones atmosféricas, caracterizado por los estados y la evolución del tiempo, en el curso de un periodo suficientemente largo, en un dominio espacial determinado (ORGANIZACIÓN METEOROLÓGICA MUNDIAL, 2011). Está condicionado por la circulación general del sistema Tierra Atmósfera a escala global; por la latitud, altura, orografía y continentalidad a escala regional, y por los usos y coberturas del suelo a escala local. En general, se estima que un período de treinta años reproduce condiciones de normalidad estadística, para cuantificar el comportamiento promedio de diferentes parámetros meteorológicos (INTERGUVERNAMENTAL PANEL ON CLIMATE CHANGE 1995).

La urbanización modifica las características físicas de la superficie terrestre, por medio de las cuales la radiación solar es capaz de ser absorbida, almacenada, transformada y emitida a la atmósfera (KUSAKA 2008). $\mathrm{Al}$ respecto, los materiales de construcción urbanos, tienen mayor inercia térmica $\mathrm{y}$ propician una mayor absorción de radiación de onda corta, y menor emisión de radiación de onda larga, por encima de los elementos de rugosidad (GRIMMOND \& OKE 1998). Consecuentemente, el enfriamiento radiativo es mucho menor en la ciudad, que en zonas rurales, ya que el calor acumulado en las estructuras urbanas, queda almacenado por varias horas tras la puesta de sol (OKE 1987). A las propiedades físicas de las coberturas del suelo, se añaden las intensidades de sus usos y consumos de energía asociados (CUADRAT et al. 1993; SARRICOLEA et al. 2008; LAMPTEY 2009; COUTTS et al. 2007), así como la configuración de los elementos sobre la superficie (OKE 1987; LINDBERG et al. 2003). Altas densidades de construcción y edificios de gran altura, separados por cañones urbanos estrechos, por ejemplo, modifican la dirección e intensidad de los flujos de viento, intervienen en el paso de la radiación solar incidente y retardan 
los tiempos en los cuales la radiación de onda larga, se libera a la atmósfera durante la noche. Todo lo anterior, define un comportamiento característico de los parámetros meteorológicos al interior de las ciudades, que dan origen a un clima urbano, el cual puede ser entendido como una perturbación local del clima regional (MONTÁVEZ et al. 2000; PAPPARELLI et al. 2011).

La isla de calor urbano, constituye un fenómeno característico del clima de las ciudades y la principal consecuencia de la modificación de las temperaturas atmosféricas (LAMPTEY 2009). Se define como la diferencia en el gradiente térmico, que existe entre áreas urbanas y su entorno rural o natural, donde la ciudad es comparativamente más caliente (OKE 1987; CUADRAT et al. 2003; ARNFIELD 2003).

Que las ciudades sean más calientes, así como la presencia de islas de calor, responde principalmente a la falta de humedad (COUTTS el al. 2007), a la admitancia térmica de los elementos de superficie urbanos (GRIMMOND \& OKE 1998), a la morfología o geometría urbana (OKE 1987; LINDBERG et al. 2003), a las altas cantidades de energía utilizadas en el desarrollo de las rutinas de las personas que habitan en ellas, especialmente en áreas céntricas,(OKE 1987; CUADRAT et al. 1993; SARRICOLEA et al. 2008; LAMPTEY 2009) y al predominio de condiciones que en general, intervienen en la movilidad y mezcla de las masas de aire, como cuencas cerradas, condiciones sinópticas que favorezcan la ocurrencia de inversiones térmicas o a la ocurrencia de tiempos meteorológicos caracterizados principalmente por vientos débiles. Lo anterior, hace que en el análisis de la variabilidad del comportamiento diario y distribución espacial de las temperaturas, así como en el análisis de la intensidad de una isla de calor, definida esta última, como la máxima diferencia observada en las temperaturas, entre el centro de la ciudad y la periferia rural en un momento determinado (CUADRAT et al. 2005; SARRICOLEA 2008), sea determinante incluir características específicas de los usos y coberturas del suelo (MOLINA 2007; COUTTS et al. 2007), sobre todo aquellas asociadas a las densidades de edificación y presencia de áreas verdes (CUADRAT et al. 2005; YUAN \& BAUER 2007; SARRICOLEA et al. 2008), rasgos geográficos que den cuenta de la orografía (CUADRAT et al. 2005) y factores culturales que determinan comportamientos, hábitos y rutinas a lo largo del día (WIENERT \& KUTTLER 2005; STEWART \& OKE 2009).

Respecto a la estacionalidad de una isla de calor, se dice en general, que su ocurrencia aumenta en el verano, debido a la presencia de condiciones meteorológicas favorables a la acumulación de calor, como cielos despejados y vientos débiles (MONTÁVEZ et al. 2000; CUADRAT et al. 2005). Por el contrario, una isla de calor puede desaparecer e inclusive no formarse si las condiciones meteorológicas son desfavorables. Estados de tiempo frecuentes en otoño e invierno, como fuertes vientos y cielos cubiertos, dificultarían su formación debido a la reducción de la radiación solar que alcanza finalmente el suelo y a la mezcla de las masas de aire urbanas y rurales (GEORGAKIS et al. 2010; SALAMANCA 2010; VERÓN 2010). No obstante, hay quienes relativizan la ocurrencia de las mismas, a una combinación más variada de factores, en donde por ejemplo la presencia de nubosidad podría perfectamente ser una condición favorable para su formación (RUIZ - FLAÑO et al. 2008).

La morfología de las islas de calor en tanto, se caracteriza por mostrar una distribución concéntrica de las isotermas, aunque en el caso de ciudades costeras, muchas veces estas se perfilan por la línea de costa quedando la isoterma central abierta en dirección al mar (CARRERAS et al. 1990). La presencia de extensas mesetas térmicas, 
es decir, vastas áreas con similar temperatura que muestran consecuentemente una alta homogeneidad en las mismas, es según el mismo autor, otra característica que se repite en un gran número de casos.

Cabe mencionar, que la temperatura atmosférica se relaciona de manera inversa con la humedad relativa, consecuentemente cuando se genera una isla de calor, también se genera una isla de sequedad (CARRERAS et al. 1990). Al respecto, la presencia de vegetación y cuerpos de agua, contribuyen de muchas formas a inhibir el deterioro ambiental de las ciudades y a controlar los extremos en ambos parámetros meteorológicos, actuando como variables determinantes además del viento, en la formación y control de la intensidad de las mismas (VILELA 2004; CUADRAT et al. 2005).

La vegetación por medio de la evapotranspiración, aumenta la humedad relativa del aire y disminuye la cantidad de energía disponible en la atmósfera para ser almacenada en las estructuras urbanas, reduce las temperaturas sobre todo en verano, y adicionalmente, el consumo de energía por concepto de calefacción y aire acondicionado. Sus sombras disminuyen hasta en un $20 \%$ la radiación solar y su follaje y densidad pueden reducir entre un 10 y $30 \%$ la velocidad del viento (BOLUND \& HUNHAMMAR 1999). Adicionalmente, fijan contaminantes atmosféricos, especialmente $\mathrm{CO}^{2}$; mejoran las condiciones de ventilación de las ciudades ya que enfrían las masas de aire que se desplazan hacia zonas con menor presión atmosférica, generando así brisas locales; reducen la velocidad de los vientos y contrarrestan la escorrentía superficial de aguas lluvias, entre otros (NOWAK \& MCPHERSON 1993; SPRONKEN - SMITH \& OKE 1999; CHIESURA 2004; VILELA 2004; ESCOBEDO et al. 2008). Su rol como regulador térmico, ha sido ampliamente estudiado, llegando a existir un vasto consenso en que las islas de calor se crean principalmente por la falta de vegetación (AKBARI et al. 2001; MOLINA 2007; SARRICOLEA et al. 2008 ), cuestión que ha llevado a que gran parte de las medidas de mitigación abocadas al control de temperaturas extremas y mejora de la calidad del aire entre otros, estén dadas justamente por la creación, densificación y gestión de área verdes (CHAO et al. 2010), destacándose que el volumen, ubicación, tipo y condición de la vegetación urbana influye directamente sobre sus efectos benéficos (NOWAK \& MCPHERSON 1993; VÁSQUEZ \& ROMERO 2007).

En el caso de la humedad del aire, hay estudios en donde se señala que una vez que el paisaje natural se sustituye, incluso con urbanización de baja densidad, la evapotranspiración se reduce significativamente, aumentando la disponibilidad de energía en el ambiente y disminuyendo la regulación de temperaturas elevadas. Considerando lo anterior, autores como COUTTS et al. (2007) destacan que las medidas de mitigación referidas al aumento de cobertura vegetal, deben ir acompañadas de estrategias de retención de agua, con el fin de aumentar la eficiencia $y$ eficacia de los servicios ambientales asociados a ellas.

Entre los efectos adversos de la modificación del clima asociados a la urbanización destaca el disconfort térmico causado por el exceso de calor y/o frío, lo que a su vez propicia, el aumento de consumo energético para climatización. Al respecto, AKBARI et al. (2001), señala que una ciudad promedio, registra durante una tarde de verano $2,5^{\circ} \mathrm{C}$ más que sus alrededores rurales, y que la demanda energética se incrementa entre un 2 y $4 \%$, por cada $1^{\circ} \mathrm{C}$ de aumento de la temperatura del aire, pasado los $20^{\circ} \mathrm{C}$ (AKBARI et al. 2001). La concentración y estabilización de gases contaminantes, sobre determinadas áreas de la ciudad, favorece el aumento en la frecuencia y severidad de enfermedades respiratorias (el ozono por ejemplo, tiene un efecto vasoconstrictor), y 
es otro de los efectos más notorios, nocivos y costosos de los climas urbanos. Este proceso, vinculado a la modificación de los flujos de aire, que tienden a confluir por diferencias de presión sobre las zonas térmicamente más cálidas y debido a la falta de ventilación, posee una relación directa con la cantidad de energía disponible en la atmósfera y por tanto, con la presencia, densidad y estado de la vegetación (NOWAK \& MCPHERSON 1993, AKBARI et al. 2001; SARRICOLEA et al. 2008).

Antecedentes importantes de considerar en la ciudad de Santiago son aquellos entregados por SARRICOLEA 2012, quien concluye que en los últimos 56 años, la temperatura media de las máximas y mínimas se ha incrementado en $1^{\circ}$ y $0,83^{\circ} \mathrm{C}$ respectivamente. Ello se debería principalmente al efecto de la isla de calor urbano que se genera al interior de ella, la cual manifiesta su mayor intensidad durante la medianoche, en las estaciones de verano y primavera bajo contextos de cielo despejado y circulación anticiclónica. De acuerdo a este autor, el $44,5 \%$ de las islas de calor analizadas presentan una distribución concéntrica de sus isotermas y las variables más influyentes en su formación son, el albedo de la superficie, el estado y presencia de la vegetación y la densidad de construcción.

Algunos estudios previos al ya descrito, mencionan además, que hay un patrón similar entre la distribución de las temperaturas atmosféricas y las de emisión superficial. Ambas se encuentran comandadas fuertemente por la distribución de los usos y coberturas del suelo (MOLINA 2007; SARRICOLEA 2008) y en el estudio de MOLINA (2007), se destaca que hay variaciones significativas de la temperatura del aire, al hacer pequeños incrementos en los porcentajes de coberturas con vegetación.

Respecto a estudios de la variabilidad térmica al interior de Valparaíso, destacan dos publicaciones. La primera, hace referencia a la variabilidad diaria y estacional, de las temperaturas atmosféricas y su relación con la condición socioeconómica de la población (ROMERO et al. 2008), mientras que la segunda, relaciona la variabilidad estacional de las temperaturas de emisión superficial en el área Metropolitana de Valparaíso con diferentes factores explicativos (SARRICOLEA \& ROMERO 2009).

En los resultados presentados por ROMERO et al. (2008), destaca durante la estación de verano, la presencia de micro islas de calor durante la mañana y de una isla de calor sobre el centro o Plan de la ciudad, durante la noche. Se observa a lo largo del día, la presencia de masas de aire frío que descienden por la quebrada Bellavista desde el sector de Quebrada Verde.

SARRICOLEA \& ROMERO (2009) en tanto, utilizan una regresión múltiple paso a paso, identificando la relación de las variables distancia al mar, elevación topográfica, exposición de laderas, presencia y estado de la vegetación (NDVI) y humedad y reflectividad del suelo, con las temperaturas de emisión superficial en la mañana. Los resultados muestran que es posible encontrar diferencias en el comportamiento de las temperaturas promedio, entre el área urbana del área Metropolitana de Valparaíso y su entorno rural, de hasta $4,4^{\circ} \mathrm{C}$ en verano y $2,8^{\circ} \mathrm{C}$ en invierno y que al considerar los valores máximos urbanos y mínimos rurales, estas diferencias aumentan a $17,4^{\circ} \mathrm{C}$ en verano y a $8,8^{\circ} \mathrm{C}$ en invierno.

Tanto Santiago como Valparaíso, han pasado por un rápido proceso de aumento de su población, con la consecuente modificación de usos y coberturas del suelo. Los efectos de dichos procesos sobre el clima local, son en general desconocidos, cuestión que es preocupante si se consideran las proyecciones entregadas en el contexto de cambio climático. La rapidez y magnitud, con la que se anticipa que ocurrirán los cambios en el sistema Atmósfera-Tierra, cuestionan 
la capacidad adaptativa de la población urbana y ponen en relieve la importancia de generar información que dé cuenta de relaciones espacial y temporalmente vinculadas entre los componentes del paisaje urbano y la variabilidad de los parámetros meteorológicos que las definen. De acuerdo a lo que señala el penúltimo INFORME SOBRE CAMBIO CLIMÁTICO (2007), la frecuencia de días calurosos aumentará, en el caso de Chile, la zona central vivirá un proceso de desertificación, y en general, la población vulnerable será la más afectada por dichos procesos. Recogiendo aquello, Santiago y Valparaíso se vuelven áreas de estudio especialmente relevantes, pues aunque no forman parte de las zonas del país con clima definido como semiárido, se localizan en un área de transición climática. En el caso de la capital, el acceso a servicios ambientales está fuertemente condicionado a la capacidad adquisitiva de sus habitantes y en Valparaíso, su población, mayoritariamente de los grupos socioeconómicos D y E (ROMERO et al. 2008), su orografía, condición de ciudad costera, y urbanización muchas veces precaria, incrementan la vulnerabilidad de las personas, así como el riesgo a la ocurrencia de desastres naturales.

Considerando lo anterior, la presente investigación ha tenido como propósito, disminuir los niveles de incertidumbre respecto al comportamiento y distribución espacial de la temperatura atmosférica en el área urbana de Santiago y Valparaíso, en un contexto de aumento persistente de población y de superficies urbanizadas. Se aborda la relación entre temperaturas y componentes naturales y artificiales del paisaje urbano, con el fin de identificar aquellas variables que puedan ser consideradas en las herramientas de gestión y planificación ambiental de ambas ciudades.

\section{MATERIALES Y MÉTODOS}

El desarrollo de la investigación, ha sido abordado mediante el análisis de las temperaturas atmosféricas, verticalmente a escala del dosel y horizontalmente a escala local, definida esta última por la mancha urbana de las ciudades. Se utilizaron datos

horarios de estaciones automáticas (Fig.1) y convencionales. 29 en el caso de Santiago (Fig. 2) y 13 en Valparaíso (Fig. 3).

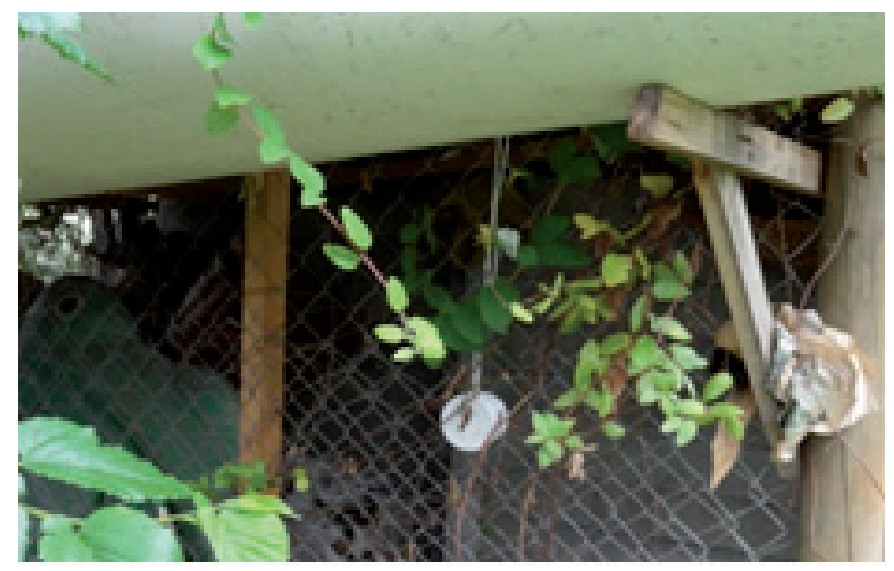

Fig.1. Localización tipo de estaciones meteorológicas automáticas. Los sensores de temperatura Hobo, fueron instalados a partir del segundo semestre del año 2008, a un metro y medio de altura, siempre a la sombra, y protegidos al interior de una caja blanca con perforaciones. Fuente: Archivo personal de las autoras.

Fig. 1. Location type Hobo temperature sensors. Were installed in the second half of 2008, to five feet high, always in the shade, and protected inside a white box with perforations. Source: 
Se evaluó la consistencia de las series por medio de un control de calidad y homogenización de datos. Para la selección de los días a analizar, se consideró en el caso de Santiago la oscilación térmica estival promedio de los datos y la disponibilidad de una imagen satelital ASTER y una imagen LANDSAT TM (4 y 12 de febrero del
2009 respectivamente). En Valparaíso en tanto, se clasificaron los días de verano en templados, cálidos y fríos, utilizando como referencia el límite inferior y superior del error típico de la media muestral y se tuvo en cuenta, la fecha de la imagen de satélite LANDSAT TM disponible para el cálculo de la presencia y estado de la vegetación (6 de Febrero del 2011).

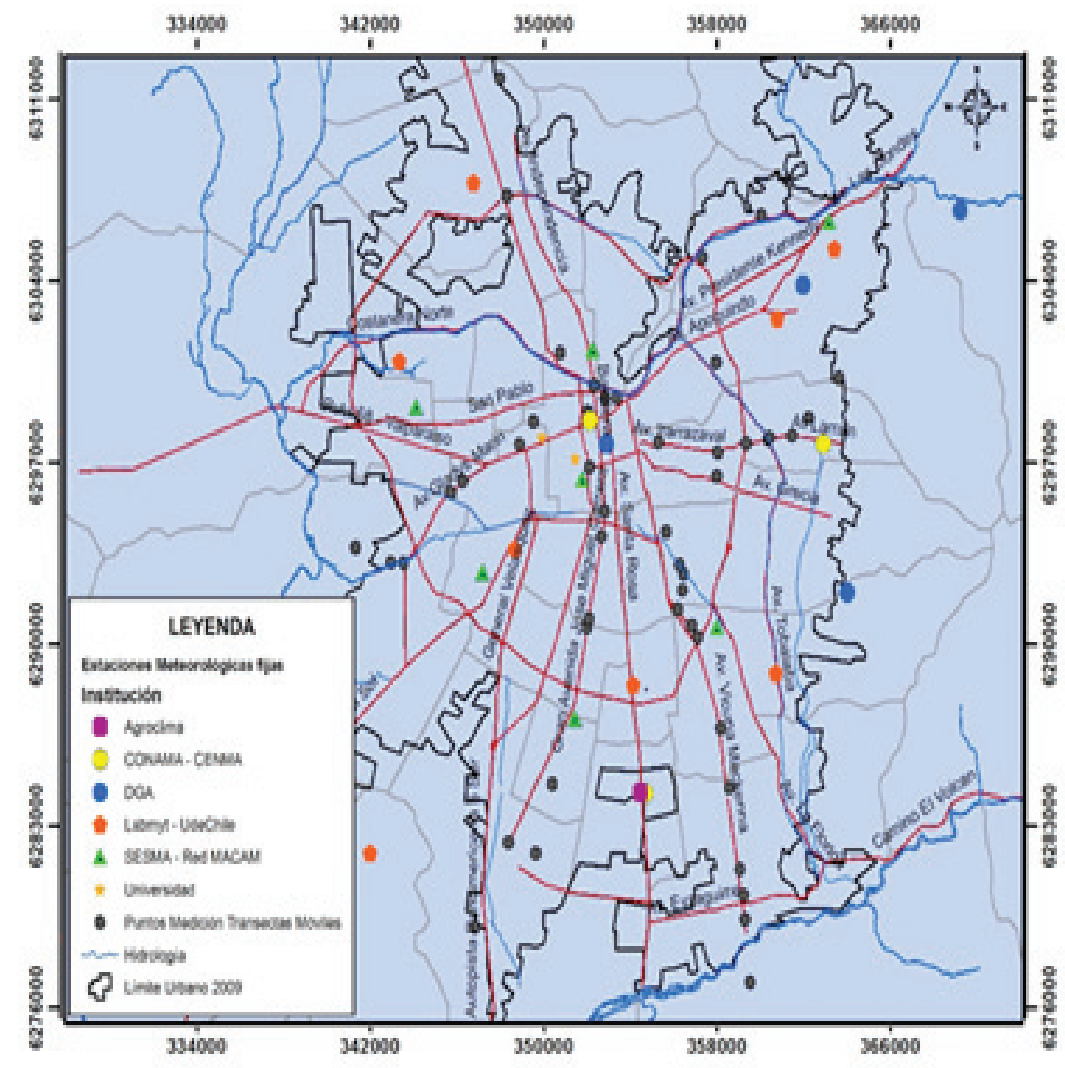

Fig. 2. Localización de estaciones meteorológicas en la ciudad de Santiago. En las mediciones móviles se consideraron seis transectos durante los días 20 y 21 de enero del 2009, a las 9:00, 14:30 y 20:00 hrs.) Fuente: Elaboración propia.

Fig. 2. Location of weather stations in the city of Santiago. Six transects were considered for 20 and 21 January 2009, at 9:00, 14:30 and 20:00 hrs on mobile measurements). Source: Authors. 


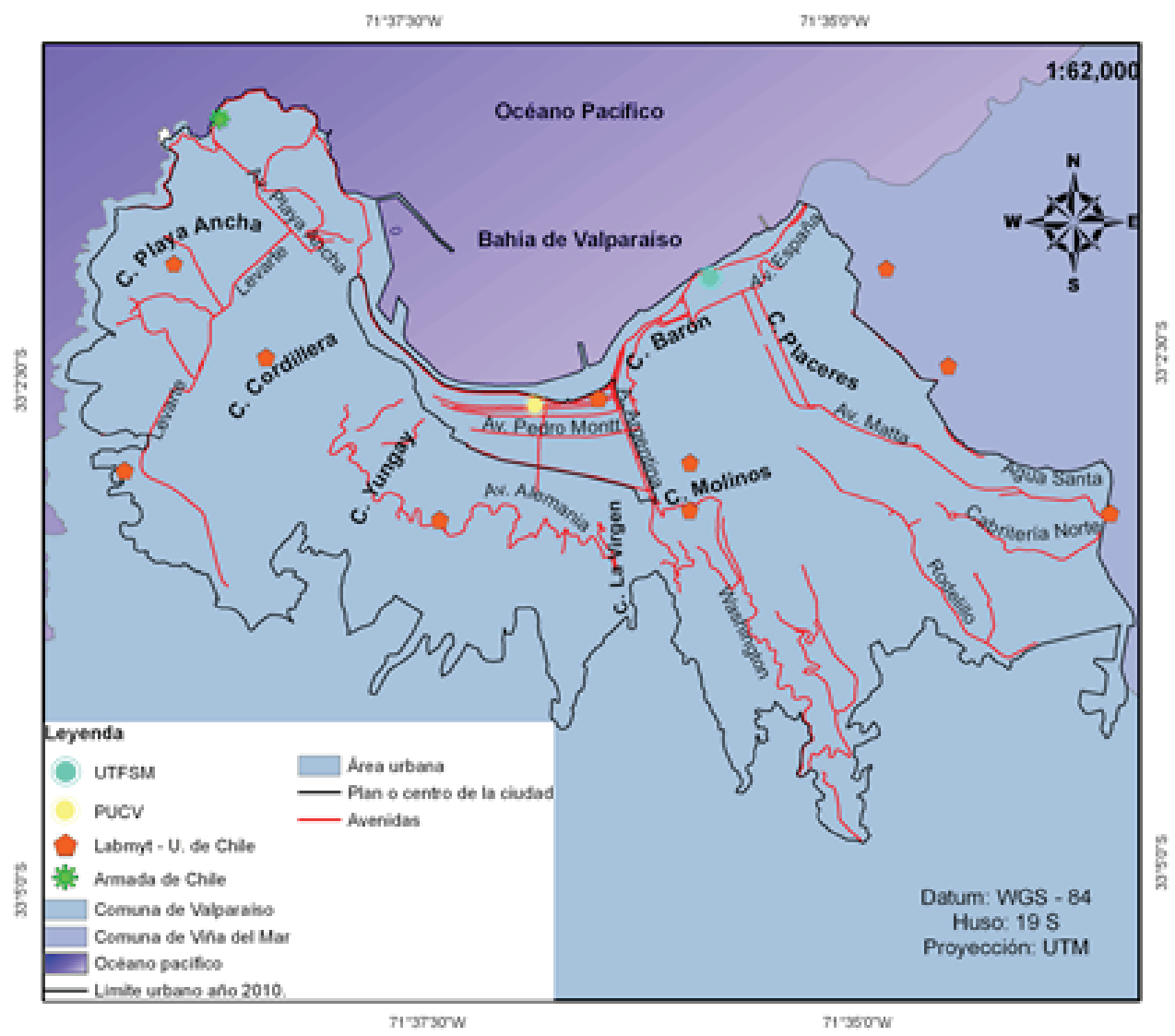

Fig. 3. Localización Estaciones Meteorológicas en la ciudad de Valparaíso. Fuente: Elaboración propia.

Fig. 3. Location meteorological stations in the city of Valparaiso. Source: Authors.

En ambos casos, se analizaron las condiciones sinópticas y meteorológicas prevalecientes, con el objetivo de descartar fenómenos meteorológicos significativos que pudiesen corresponder a una situación anómala (Fig. 4). Finalmente, los días escogidos como representativos del clima urbano durante la estación de verano, fueron el 21 de enero del 2009 en Santiago y el 01 de febrero del 2011 en Valparaíso. 


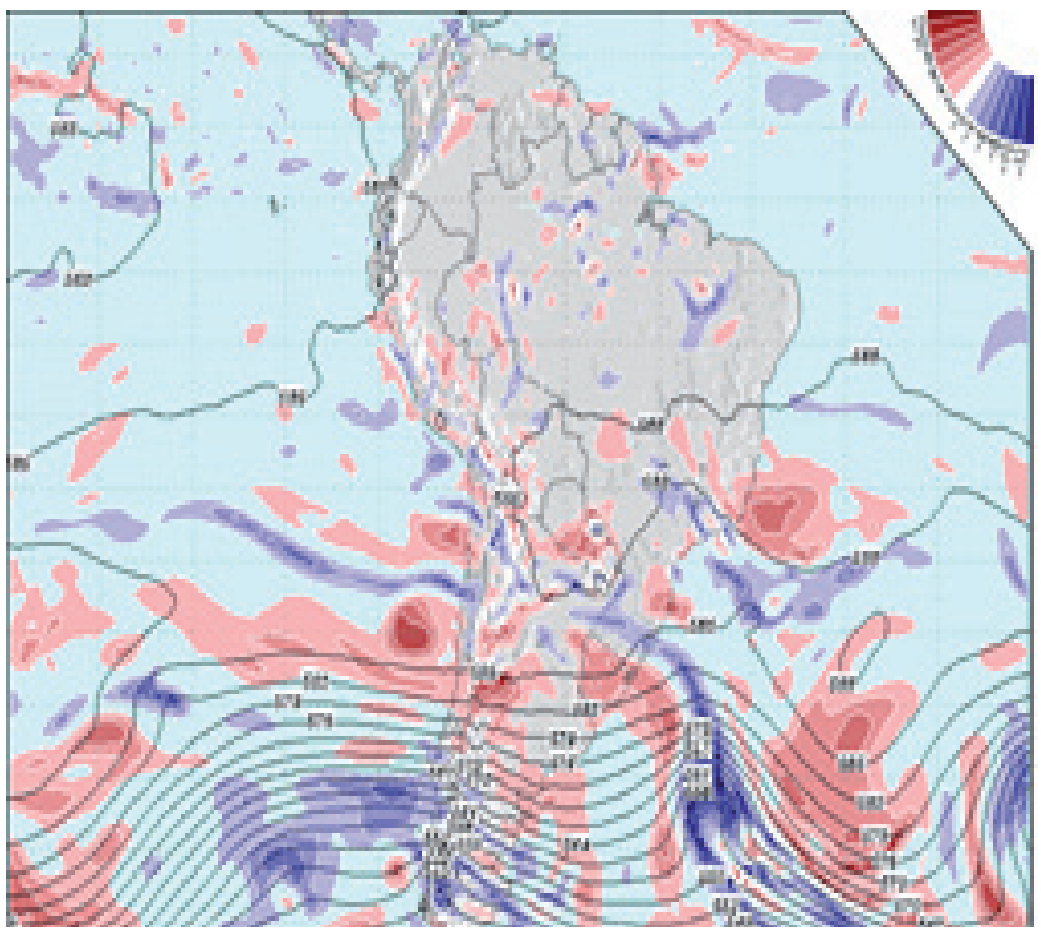

Fig. 4. Carta de presiones atmosféricas a los 500 hPa del 1 de Febrero del 2011. Para analizar las condiciones sinópticas, se contó con una carta de presiones para cada día evaluado en las ciudades de Santiago y Valparaíso. Fuente: CENTRO NACIONAL DEL MEDIOAMBIENTE (CENMA 2011).

Fig. 4. Letter from atmospheric pressures to $500 \mathrm{hPa}$ of February 1, 2011. To analyze the synoptic conditions, included a letter for every day evaluated in the cities of Santiago and Valparaiso. Source: NATIONAL ENVIRONMENTAL CENTER (CENMA 2011).

La representación espacial de las temperaturas atmosféricas, se hizo utilizando el interpolador Kriging ordinario, el cual asigna pesos a los puntos muéstrales, en función de la dirección y la distancia con otros puntos conocidos (EASTMAN 2003).

Para el análisis explicativo del comportamiento y distribución espacial de las temperaturas, se identificaron variables que son significativas en el ámbito del clima urbano según la literatura, pero que además dan cuenta de componentes naturales y artificiales que caracterizan la especificidad del paisaje de las ciudades y que por tanto, debieran estar estrechamente vinculados a la variabilidad de sus temperaturas atmosféricas y clima en general. Estas tienen relación con la orografía, la morfología urbana, la intensidad de los usos de suelo y la presencia de humedad en el aire. Los materiales utilizados fueron coberturas digitales preexistentes, un mosaico de fotografías aéreas del año 2010, proporcionado por la Secretaría de Planificación y Transporte (SECTRA) e 
imágenes satelitales estivales, captadas el04 (ASTER) y 12 (LANDSAT) de febrero del 2009 en Santiago, y el 06 de Febrero del 2010en Valparaíso.Todos fueron procesados en los Sistemas de Información Geográfica, ArcGis 10 e Idrisi Taiga versión 16.
Las variables seleccionadas, los autores que han sido tomados como referencia, y el método aplicado para la espacialización de las mismas, se describen en la tabla 1.

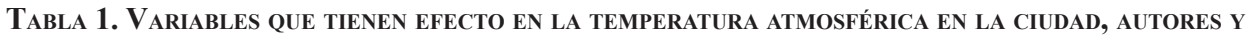
METODOLOGÍAS UTILIZADAS PARA SU CÁLCULO Y REPRESENTACIÓN ESPACIAL.

\begin{tabular}{|c|c|c|}
\hline VARIABLE & AUTORES & METODOLOGÍA \\
\hline $\begin{array}{l}\text { Distancia a cursos de agua / } \\
\text { Distancia al mar (Valparaíso) }\end{array}$ & $\begin{array}{l}\text { PIGEON et al. 2007; RUIZ- } \\
\text { FLAÑO et al. 2008; MENDONCA } \\
2009\end{array}$ & Mínima distancia lineal \\
\hline $\begin{array}{c}\text { Presencia y estado de la } \\
\text { vegetación / Cobertura vegetal } \\
\text { (Santiago) }\end{array}$ & $\begin{array}{l}\text { OKE 1987; YUAN \& BAUER } \\
\text { 2007; COUTTS et al. 2007; } \\
\text { HONJO et al. 2003; } \\
\text { KARDINAL 2008 }\end{array}$ & Teledetección \\
\hline $\begin{array}{c}\text { Distancia a formaciones de } \\
\text { vegetación densa }\end{array}$ & $\begin{array}{c}\text { NOWAK \& MCPHERSON 1993; } \\
\text { ZHANG et al. } 2008\end{array}$ & Fotointerpretación \\
\hline $\begin{array}{l}\text { Proporción de superficie } \\
\text { impermeabilizada }\end{array}$ & $\begin{array}{c}\text { ARNOLD \& GIBBONS 1996; } \\
\text { YUAN \& BAUER 2007; ZHANG } \\
\text { et al. } 2008\end{array}$ & $\begin{array}{l}\text { Fotointerpretación ZCT / } \\
\text { Teledetección (Santiago) }\end{array}$ \\
\hline Elevación topográfica & $\begin{array}{c}\text { JO et al. 2001; WIENERT \& } \\
\text { KUTTLER 2005; SARRICOLEA \& } \\
\text { ROMERO } 2009\end{array}$ & $\begin{array}{c}\text { Interpolación de Delaunay } \\
\text { (TIN) }\end{array}$ \\
\hline Exposición de laderas & SARRICOLEA \& ROMERO 2009 & $\begin{array}{c}\text { Interpolación de Delaunay } \\
\text { (TIN) }\end{array}$ \\
\hline Densidad de población & $\begin{array}{l}\text { OKE 1987; WIENERT 2005; } \\
\text { SARRICOLEA } 2008\end{array}$ & Densidad por hectárea \\
\hline Proporción de superficie construida & $\begin{array}{l}\text { ARNFIELD 2003; PAULEIT et al. } \\
\text { 2005; ZHANG et al. } 2008\end{array}$ & Fotointerpretación ZCT \\
\hline Flujo de calor antropogénico & $\begin{array}{c}\text { GRIMMOND \& OKE 1998; } \\
\text { GUIJARRO 1998; WIENERT } 2005\end{array}$ & Fotointerpretación ZCT \\
\hline Rugosidad & \multirow{3}{*}{$\begin{array}{l}\text { SPRONKEN - SMITH \& OKE } \\
\text { 1999; ELIASSON 1999; HONJO } \\
\text { 2003; LINDBERG et al. 2003; } \\
\text { ARNFIELD 2003; } \\
\text { GRIMMOND } 2007\end{array}$} & \multirow{3}{*}{$\begin{array}{c}\text { Fotointerpretación ZCT/ } \\
\text { Altura media por manzana } \\
\text { (Santiago) }\end{array}$} \\
\hline Altura media edificaciones & & \\
\hline Factor de visibilidad del cielo & & \\
\hline Temperatura de emisión superficial & ARNFIELD 2003; PRASHAD 2004 & Teledetección \\
\hline
\end{tabular}

Fuente: Elaboración Propia.

Para la selección de las variables explicativas, se evalúo cada una de ellas respecto de la temperatura atmosférica a través de una correlación bivariada entre pares de variables, utilizando los coeficientes de Pearson y Spearman previo análisis de la 
normalidad de los datos. Posteriormente,se aplicó una regresión por mínimos cuadrados ordinarios, para determinar el grado de dependencia simultánea de la temperatura con las variables explicativas que poseían una relación estadísticamente significativa con la temperatura. En base a ello se construyeron las ecuaciones de regresión múltiple en el programa de estadística SPSS Versión 18. Se realizaron modelos para la mañana, tarde y noche, identificando de este modo, la variabilidad espacial de las temperaturas durante la mañana, tarde y noche. Momentos del día en que se registran las primeras horas de calor, la máxima insolación, y la puesta de sol respectivamente.

\section{RESULTADOS}

\section{Comportamiento y distribución espacial de las temperaturas atmosféricas en la ciudad de Santiago}

La ciudad de Santiago presenta importantes $\mathrm{y}$ significativas diferencias térmicas en su interior y zonas rurales, que permiten caracterizar islas de calor y frío, que conforman verdaderos archipiélagos térmicos expresados como gradientes de variabilidad, continuos y discretos.

Durante el día el área urbana no es térmicamente homogénea, las áreas más frías se encuentran localizadas principalmente en el sector oriente de la ciudad y el sector más cálido se concentra en el sector sur. El oriente posee temperaturas similares a las zonas rurales. En términos generales la temperatura decrece del centro a la periferia, fuera de la circunvalación de Américo Vespucio. Es posible identificar el cono de aproximación del ex aeropuerto de Cerrillos y la existencia de algunos parches de características agrícolas hacia el sur; sin embargo, las temperaturas en Pudahuel, cercanas al aeropuerto internacional, localizadas fuera del límite urbano, describen islas de calor que aumentan su superficie de la mañana a la tarde. Es posible identificar el Parque O'Higgins y la Quinta Normal como áreas de menor temperatura. La Quinta Normal alcanza hasta $3^{\circ} \mathrm{C}$ de diferencia con la matriz urbana circundante y el Parque $\mathrm{O}^{\prime}$ Higgins es $2^{\circ} \mathrm{C}$ más frío.

Unas horas después de la puesta de sol, Santiago se convierte en una gran y única isla de calor urbana que incluye casi por completo la superficie urbana, existiendo zonas cálidas también en las áreas rurales ubicadas al norte. La isla de calor urbana se propaga desde el centro hacia el oriente de la ciudad (Fig.5). La diferencia que existe entre los sectores más cálidos y los más fríos, y su entorno no urbano, alcanzan los $12^{\circ} \mathrm{C}$.

En este horario, la distribución térmica es relativamente homogénea, ya no es posible observar la distinción oriente - poniente, $\mathrm{ni}$ se perciben las diferencias entre las áreas vegetadas y las construidas. La ciudad ha acumulado gran cantidad de calor durante el día, con temperaturas superiores a $30^{\circ} \mathrm{C}$ por varias horas y comienza luego de la puesta de sol, a devolver lentamente el calor acumulado a la atmósfera. La vegetación no logra tener un efecto significativo a esta hora y a esta escala.

El efecto de las áreas rurales del sur de la ciudad es importante a esta hora, ingresan temperaturas menores por el cono de aproximación del aeropuerto de Cerrillos y en partes de la comuna de La Pintana y Puente Alto. Esta diferencia respecto a los sectores rurales del poniente y norte, puede deberse al predominio de la actividad rural en los primeros y a la presencia del Río Maipo, que permitiría una circulación mayor de vientos. Las áreas rurales del norte y poniente por el contrario, han sido paulatinamente urbanizadas constituyendo islas de calor urbanas. En ellas, se localiza gran parte de las industrias, el aeropuerto internacional y suelos que corresponden a un depósito de cenizas. 


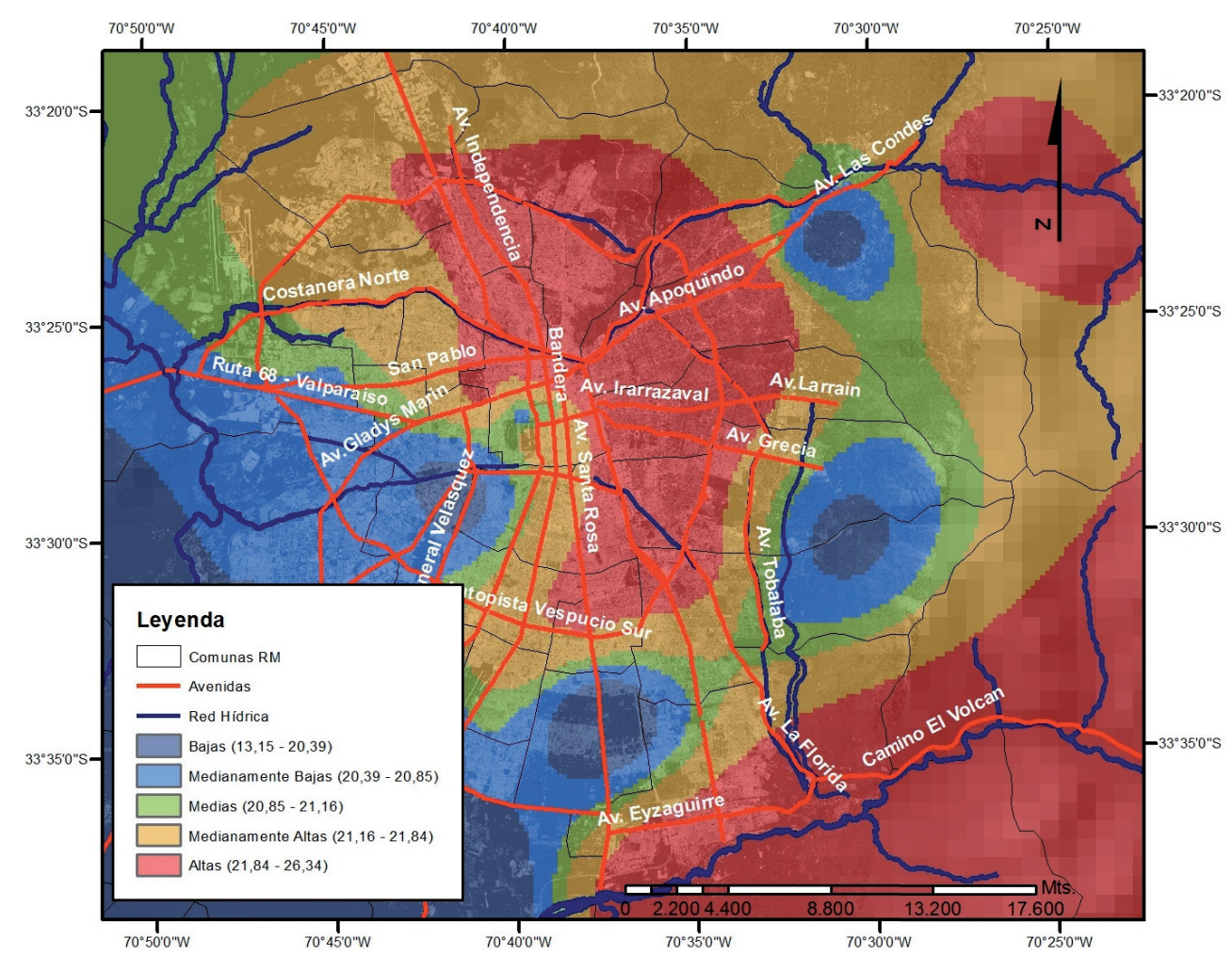

Fig.5. Distribución espacial de las temperaturas atmosféricas a las 22:00 hrs, en la ciudad de Santiago. Fuente: Elaboración propia.

Fig. 5. Spatial distribution of atmospheric temperatures at 22:00 hrs, in the city of Santiago. Source: Authors.

Comportamiento y distribución espacial de las temperaturas atmosféricas en la ciudad de Valparaíso

La variabilidad horaria de la temperatura durante el 1 de Febrero, muestra en sus registros un aumento promediode $1,5^{\circ} \mathrm{C}$ (en el caso de los más bajos) y $2,3{ }^{\circ} \mathrm{C}$ (en el caso de los más altos) cada tres horas. En el caso de las temperaturas más elevadas, se observa que su comportamiento durante la mañana y noche es muy similar, mostrando su mayor variabilidad entre las 9:00 y 12:00 y las 18:00 y 21:00 hrs. En el caso del primer intervalo, el aumento de la mínima es de sólo $0,1^{\circ} \mathrm{C}$, y el de la máxima de $3,1^{\circ} \mathrm{C}$; en el caso del segundo, la mínima desciende $2,8^{\circ} \mathrm{C}$, mientras que la máxima lo hace en $8,3^{\circ} \mathrm{C}$. La máxima diaria ocurre a las 18:00 hrs, y en general, se observa que la variabilidad de las temperaturas más elevadas es mayor a la de las temperaturas más bajas. La diferencia en los registros, en distintas áreas de la ciudad durante un mismo horario, es alta a excepción de la noche, y bordea los $11^{\circ} \mathrm{C}$ durante las horas de mayor insolación.

Durante la mañana (9:00 hrs), la diferencia entre las temperaturas más y menos elevadas alcanza ya $\operatorname{los} 10,5^{\circ} \mathrm{C}$. El norte de la ciudad, es gradualmente más frío que el sur, no se distinguen islas o micro islas de calor o frío y en general, la variabilidad espacial de la temperatura es baja.

En la tarde (15:00 hrs), la diferencia entre las temperaturas más y menos elevadas llega a $\operatorname{los} 11,5^{\circ} \mathrm{C}$. Las temperaturas más elevadas (entre $24,1^{\circ}$ y $27,9^{\circ} \mathrm{C}$ ), se extienden a lo largo de casi toda la ciudad, tanto al oriente, centro y poniente. Áreas densamente 
urbanizadas, como las que se encuentran entre los cerros Barón y Molinos; La Virgen y Cordillera, el Plan o centro y el sector alto de Placeres y Playa Ancha, son especialmente cálidas y la inflexión de las isotermas, pareciera mostrar una gran isla de calor que va más allá de los límites de la ciudad. El extremo norte del cerro Playa Ancha y los sectores de Las Torres, La Isla y aeropuerto, al sur, en tanto, son áreas frescas, fluctuando sus temperaturas entre $16,4^{\circ}$ y $21,4^{\circ} \mathrm{C}$. El descenso de los registros, desde áreas cálidas a frías, es alto a pocos metros de distancia.
En la noche (21:00 hrs), la diferencia entre los lugares más cálidos y más fríos decrece rápidamente llegando a $\operatorname{los} 5,5^{\circ} \mathrm{C}$. Las temperaturas más elevadas, se distribuyen al igual que las 15:00 hrs. sobre gran parte de la ciudad cubriendo las áreas más densamente urbanizadas. Sin embargo esta vez, las isotermas adquieren forma de cuña abierta en dirección al mar, desde donde decrecen gradualmente las temperaturas hacia el interior (Fig.6). Los registros más bajos (entre $16,8^{\circ}$ y $19^{\circ} \mathrm{C}$ ), se concentran aisladamente esta vez, solo en los sectores de El Vergel, Las Torres, La Isla, parte alta de Rodelillo y aeropuerto.

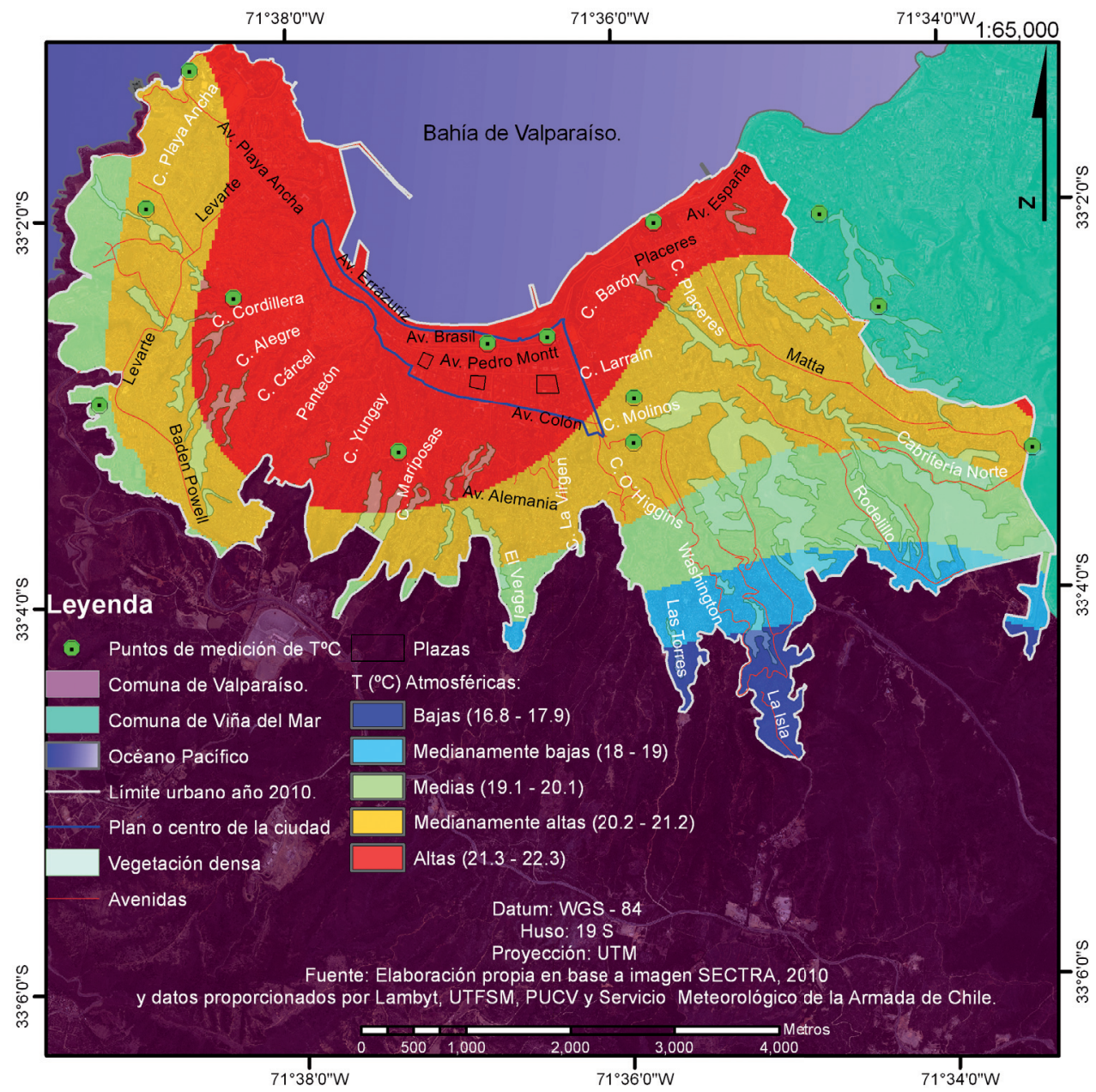

Fig. 6. Distribución espacial de las temperaturas atmosféricas a las 21:00 hrs, en la ciudad de Valparaíso. Fuente: Elaboración propia.

Fig. 6. Spatial distribution of atmospheric temperatures at 22:00 hrs, in the city of Valparaíso. Source: Authors. 
En general a lo largo del día, destacan como áreas persistentemente más frescas, el barrio universitario al norte de Playa Ancha; El Vergel, Las Torres y La Isla hacia la periferia sur, y Rodelillo y aeropuerto, al oriente. Las temperaturas de intensidad media dominan gran parte de Valparaíso hasta el mediodía, pero a las 15:00 hrs. ya se identifica una gran meseta térmica que da cuenta de una ciudad homogénea y predominantemente caliente. Durante la noche en tanto, se registra una amplia isla de calor urbano, que sigue el patrón de distribución típico de islas de calor de ciudades costeras.

Análisis de la relación entre temperaturas atmosféricas de verano y variables geográficas y urbanas.

Considerando el valor de los Coeficientes de Correlación, así como la presencia de colinealidad entre variables, se obtuvieron los modelos de regresión que se muestran en la Tabla 2. A partir de ella, es posible establecer que hay variables que favorecen el incremento y descenso de las temperaturas, conforme se incrementan sus valores.

En la ciudad de Santiago, las variables que poseen una relación estadística significativa con la temperatura atmosférica son la elevación topográfica, temperatura de emisión superficial, superficie impermeable, cobertura vegetal, y la interpolación de temperatura atmosférica realizada a las $22 \mathrm{hrs}$. La elevación topográfica queda excluida, al igual que la temperatura de emisión superficial, por corresponder a un valor que el satélite capta solo durante la mañana. Las variables que incrementan las temperaturas son la proporción de superficie impermeabilizada en todos los horarios, mientras que aquellas que la disminuyen son la vegetación, elevación topográfica y temperatura de emisión superficial en la mañana y tarde, y la vegetación en la noche.

En el caso de Valparaíso, las temperaturas se elevan cuando se incrementa el porcentaje de superficie construida a las 12:00 hrs. y con la exposición de laderas a las 15:00 y 18:00 hrs. mientras que disminuyen a las 9:00, 15:00 y 18:00 hrs. cuando aumenta el índice de diferencia vegetal normalizada y la densidad de población por hectárea, a las 9:00 y 18:00 hrs. Se incrementan también, con la distancia a formaciones de vegetación densa a las 12:00 hrs; la elevación topográfica a las 12:00 y 15:00hrs; la altura media de las edificaciones a las 15:00 hrs; la superficie impermeabilizada a las 18:00 hrs. y al variar la exposición de laderas, en el caso de la noche.

Destacan los coeficientes beta asociados al índice de diferencia vegetal normalizada, los cuales auspician una disminución considerable de las temperaturas atmosféricas, en función de la presencia y estado de la vegetación en ambas ciudades. 
TABLA2. ECUACIONES DE REGRESIÓN MÚLTIPLE, QUE EXPLICAN Y PREDICEN EL COMPORTAMIENTO Y distribución de las temperaturas, en las CiUdades de Santiago y Valparaíso

\begin{tabular}{|c|c|c|c|}
\hline HORA & CIUDAD & MODELO DE REGRESIÓN & $\mathrm{R} 2$ \\
\hline \multirow{2}{*}{ Mañana } & Santiago & $\begin{array}{c}\text { Temperatura del aire }\left({ }^{\circ} \mathrm{C}\right)=-(\text { Elevación * } 0,001)-(\text { Vegetación * } \\
0,005)+(\text { Impermeabilización * } 0,006)+(\text { Interpolación * } 1,424)- \\
\left(\text { Temperatura Superficial }{ }^{*} 0,077\right)-9,923\end{array}$ & 0,873 \\
\hline & Valparaíso & $\begin{aligned} \text { Temperatura del aire }\left({ }^{\circ} \mathrm{C}\right)= & \left(\mathrm{NDVI}{ }^{*}-14,777\right)+(\text { Densidad * }-0,016) \\
& +20,282\end{aligned}$ & 0,729 \\
\hline \multirow{2}{*}{ Tarde } & Santiago & $\begin{array}{c}\text { Temperatura del aire }\left({ }^{\circ} \mathrm{C}\right)=-(\text { Elevación * } 0,001)-(\text { Vegetación * } \\
0,015)+(\text { Impermeabilización * } 0,004)+(\text { Interpolación * 1,272) }- \\
(\text { Temperatura. Superficial * } 0,006)-8,269\end{array}$ & 0,969 \\
\hline & Valparaíso & $\begin{array}{c}\text { Temperatura del aire }\left({ }^{\circ} \mathrm{C}\right)=\left(\text { Elevación }{ }^{*}-0,025\right)+(\text { Exposición * } 0,012) \\
+\left(\mathrm{NDVI}^{*}-24,659\right)+\left(\text { Altura edificios }{ }^{*}-0,267\right)+29,341\end{array}$ & 0,914 \\
\hline \multirow{2}{*}{ Noche } & Santiago & $\begin{array}{c}\text { Temperatura del aire }\left({ }^{\circ} \mathrm{C}\right)=-(\text { Vegetación * } 0,0058)+(\text { Impermeabili- } \\
\text { zación * } 0,0064)+(\text { Interpolación * } 1,019)-0,435\end{array}$ & 0,997 \\
\hline & Valparaíso & $\begin{aligned} \text { Temperatura del aire }\left({ }^{\circ} \mathrm{C}\right)= & (\text { Exposición de laderas * }-0,007)+ \\
& 21,212\end{aligned}$ & 0,661 \\
\hline
\end{tabular}

Fuente: Elaboración propia.

A partir de los modelos es posible estimar cuantitativamente el efecto de las variables, en el aumento y/o descenso de la temperatura atmosférica. Se observan algunas similitudes durante el día. En ambas ciudades la vegetación actúa disminuyendo la temperatura, con máximos que oscilan durante el día entre $0,5^{\circ}$ y $1,5^{\circ} \mathrm{C}$ en Santiago y entre $1,4^{\circ}$ y $2,4^{\circ}$ en Valparaíso. Igualmente, la elevación topográfica disminuye el calor registrado. Por el contrario, las superficies impermeables aumentan la temperatura, aunque existen diferencias durante el día respecto a su efecto. Durante la tarde la diferencia máxima de temperatura entre un sector completamente cubierto de vegetación, respecto a un terreno $100 \%$ impermeabilizado, puede alcanzar casi los dos grados en la ciudad de Santiago y hasta 5 grados en Valparaíso. En esta ciudad, se observa que el incremento en 10 pisos de los edificios, propicia un descenso en los registros de hasta $2,7^{\circ} \mathrm{C}$, y en el caso de la exposición de laderas, estos se incrementan cuando la exposición es norte y disminuyen cuando es sur, en un promedio de $1.6^{\circ}$ y $0.5^{\circ} \mathrm{C}$ respectivamente.

\section{DISCUSIÓN}

En la ciudad de Valparaíso, la variabilidad horaria de las temperaturas es alta y los registros muestran diferencias para un mismo horario que promedian los $11{ }^{\circ} \mathrm{C}$, siendo esta diferencia más grande en las horas de mayor insolación. Lo anterior, da cuenta de cómo los distintos contextos geográficos y urbanísticos que caracterizan a la ciudad determinan comportamientos térmicos altamente heterogéneos a escala del dosel, tal como indican ARNFIELD (2003); MOLINA (2007); STEWART \& OKE (2009), SALAMANCA (2010) y OKE (2010). En el caso de la distribución espacial de las temperaturas, estos mismos autores, señalan que su variabilidad también debería ser alta a la escala trabajada, sin embargo, ello no es posible de constatar a partir de los resultados, pues la representación gráfica de las temperaturas, en todos los horarios, muestra en general una tendencia al incremento o descenso paulatino de las temperaturas de un sector a otro de la ciudad, sin poder identificarse la presencia de microislas de calor y/o frío, o una 
configuración que dé cuenta clara, de la relación de las temperaturas con variables locales, aun cuando los modelos explicativos así lo señalan. Lo anterior se atribuye a limitaciones del interpolador utilizado, cuestión que es discutida más adelante.

En la ciudad de Valparaíso las áreas persistentemente más frías concuerdan con sectores caracterizados por su exposición a vientos sinópticos como es el caso del barrio universitario de Playa Ancha y con urbanización rodeada de abundante vegetación, como El Vergel, Las Torres, La Isla, Rodelillo y aeropuerto. En la ciudad de Santiago coinciden con el aeródromo Cerrillos y su cono de aproximación, el cual permite el ingreso de los vientos provenientes del sur oeste y del sector del piedmont de la ciudad, el que posee una mayor proporción de vegetación. Esto, entrega importantes indicios de que lo que señalan muchos autores respecto al viento, como MONTÁVEZ et al. (2000), CUADRAT et al. (2005), GEORGAKIS et al. (2010), y VERÓN (2010), es correcto, y constata por otra parte, lo que NOWAK \& MCPHERSON (1993), CHIESURA (2004), VILELA (2004) y ESCOBEDO et al. (2008) entre otros, afirman en el caso de la vegetación. Ambas variables, son determinantes en la regulación de las temperaturas elevadas y contribuyen a la conformación de áreas frías al interior de la ciudad, lo que destaca la necesidad de incorporar mediciones de viento, teniendo en cuenta la condición de ciudad costera de Valparaíso y considerando algunos antecedentes para la ciudad de Santiago, los que permiten establecer que existe relación entre la dirección del viento y la distribución espacial de la temperatura atmosférica (ROMERO \& OPAZO 2010). La localización de la ciudad en una cuenca cerrada, rodeada por cadenas montañosas genera un sistema de vientos anticiclonales débiles que soplan del SW y S, lo que explicaría el efecto que se manifiesta sobre todo durante la mañana y la tarde con el cono de aproximación del ex aeropuerto de Cerrillos.
La localización de las áreas más cálidas en tanto, es mucho más diversa. Sin embargo durante la tarde y la noche, los resultados de la interpolación y de los modelos explicativos, indican que estas se corresponden con áreas densamente urbanizadas, en las cuales variables asociadas al diseño urbano y a la orografía, toman relevancia. Lo anterior respalda lo sugerido por OKE (1987), CUADRAT et al. (2003), YUAN \& BAUER (2007), SARRICOLEA et al. (2008) y LAMPTEY (2009).

Destaca a las 15:00 hrs. en Valparaíso y a las 22:00 hrs. en Santiago, la presencia de una vasta meseta térmica que se expande más allá de los límites urbanos. La magnitud de su extensión, da cuenta de una alta homogeneidad de las temperaturas predominantemente altas, lo que para CARRERAS et al. (1990) es un rasgo distintivo de las islas de calor urbanas.

A las 21:00 hrs. en Valparaíso, los resultados revelan la presencia de una isla de calor intraurbana nocturna, que muestra importantes similitudes con aquella identificada por ROMERO et al. (2008). La morfología coincide con el modelo típico de islas de calor de ciudades costeras, descrito por CARRERAS et al. (1990), según el cual, las isotermas se perfilan siguiendo la forma de la línea de costa, quedando la isoterma central abierta en dirección al mar. La temperatura disminuye en intensidad, de forma regular desde el centro a la periferia, su núcleo coincide con la localización de las áreas más densamente urbanizadas, dadas por el centro e inmediaciones, y la quebrada Yolanda, constituye un corredor desde el cual descienden masas de aire frío, las que ocasionan la inflexión de la isoterma central, en dirección al mar por el Oriente. Contrastes importantes de destacar, son la mayor variabilidad de la distribución espacial de las temperaturas, en el caso de la isla de calor urbana observada el año 2008, lo cual puede ser explicado en razón del uso de metodologías diferentes. 
Esta investigación demuestra lo planteado por ELIASSON (1999); ROHINTON (1999) Y HONJO (2003), entre otros autores, quienes establecen que en la ciudad son múltiples los factores que actúan sobre elcomportamiento meteorológico y en consecuencia, en la formación de un clima diferente.

Variables asociadas a la presencia de humedad en el aire, son las que intervienen en la variabilidad de las temperaturas de manera más drástica, cuestión que avala lo indicado por COUTTS et al. (2007). La vegetación, como concuerdan NOWAK \& MCPHERSON (1993), SPRONKEN-SMITH \& OKE (1999), CHIESURA (2004), YUAN \& BAUER (2007), MOLINA (2007) \& SARRICOLEA et al. (2008) entre muchos otros, reduce significativamente la temperatura, mientras que las coberturas que impiden la infiltración y retención de agua, la incrementan.

Los rasgos geográficos vinculados al relieve, como ha destacado CUADRAT et al. (2005), WIENERT \& KUTTLER (2005) y RUIZ-FLAÑO et al. (2008), determinan el clima local. En Valparaíso, las variables que dan cuenta de la orografía, son significativas a lo largo de casi todo el día (a excepción de la mañana) y aunque sus efectos sobre la temperatura, son mucho más moderados que aquellos asociados a la urbanización, son de suma relevancia. En esta ciudad, se constata además, lo señalado por OKE (1987), SPRONKEN-SMITH \& OKE (1999), LINDBERG et al. (2003), ARNFIELD (2003) y GRIMMOND (2007) respecto al rol de la morfología urbana en la variabilidad de las temperaturas. Sectores caracterizados por un diseño urbano en donde predominan los edificios de altura, pueden ser hasta $2,7^{\circ} \mathrm{C}$ más fríos que aquellos sectores en donde los edificios son más bajos. El horario en que esta variable aparece como significativa, indica que el descenso en la temperatura tiene relación con el menor acceso de luz solar durante el día y con el efecto de las sombras. En el caso de la exposición de laderas, se observa que estas favorecen tanto el aumento como descenso de las temperaturas dependiendo ello de la hora del día, tal como señalan SARRICOLEA \& ROMERO (2009).

Respecto a la metodología utilizada, es posible señalar que si bien, el kriging ordinario permite obtener errores de predicción más pequeños que otros interpoladores, al considerar no solo la distancia de un punto al valor muestral, sino que también, la correlación espacial que existe entre los valores de los puntos, considerando además la dirección, no permite incorporar variables que dan cuenta de la especificidad de los distintos paisajes identificados al interior de ambas ciudades. En materia de clima urbano, se reconoce y los resultados así lo confirman, que la temperatura atmosférica es producto de relaciones múltiples, es decir, su variabilidad está fuertemente influenciada por la acción conjunta de muchos otros factores, que pueden cambiar substancialmente a lo largo del día y de un lugar a otro. La correlación entre distancia y dirección de los puntos de medición, por tanto, resulta una medida insuficiente para la representación espacial de las temperaturas, pudiendo incluso alterarla, al utilizar un criterio de diferenciación, que no se corresponde necesariamente con las variables o factores que realmente determinan la variabilidad espacial en un área u otra. Esto es especialmente importante, pues puede llevar a hacer suposiciones sesgadas y/o erradas. Considerando lo anterior, la representación espacial de la temperatura, requiere de metodologías que tengan la versatilidad de incorporar variables que sean representativas de distintos contextos geográficos y urbanísticos. En el caso de Valparaíso, los modelos explicativos, muestran que la presencia y estado de la vegetación, proporción de superficies construidas, altura promedio de las edificaciones, elevación topográfica y exposición de laderas, son factores que deben ser considerados en futuros análisis espaciales del campo térmico de la ciudad. En el mismo sentido, para el caso de Santiago resulta necesaria la consideración de la cobertura de vegetación, la proporción de superficies construidas, la elevación topográfica y temperatura de emisión superficial. 
En este escenario, el uso de estadística inferencial, permite no solo explicar la variabilidad térmica de las temperaturas, sino que también, en un futuro podría permitir representarlas espacialmente de manera más fidedigna. Presenta la ventaja además, de ser una metodología en la cual tiene cabida en cada paso metodológico, el criterio de quien la utiliza, lo que resulta muy útil para identificar sesgos en los modelos, cuando el tamaño de las muestras por ejemplo, limita la representación de determinadas variables.

En cuanto al uso futuro de estadística inferencial para la representación espacial de las temperaturas atmosféricas, es preciso hacer mención a la necesidad de incrementar las mediciones de temperatura a escala del dosel. $\mathrm{Al}$ respecto, los resultados expuestos, indican que en la localización de nuevas estaciones, deberían considerarse las variables señaladas y dada la importancia que los modelos dan a la orografía, es que correspondería considerar a las cuencas hidrográficas como las unidades ambientales estructurantes. Esto requiere de un número de estaciones que en conjunto, logren dar cuenta del comportamiento térmico de las cuencas al menos más representativas, y que permitan validar a la vez, los resultados.

\section{CONCLUSIONES}

Esta investigación reconoce aquellos factores que influyen en la formación del clima urbano. De los facores considerados a partir de la literatura, sólo cinco son finalmente aquellos que se relacionan con el comportamiento de la temperatura atmosférica. Las variables que se seleccionaron para ser introducidas en los modelos de regresión, varían para cada ciudad y en cada una de ellas también varían durante el día. Por lo tanto, el comportamiento de la temperatura permite ser explicado por diferentes factores locales en la mañana, tarde y noche.

Los factores que coinciden en ambas ciudades son la vegetación, la impermeabilización y la elevación topográfica, sin embargo, sólo la vegetación y la altura presentan una influencia similar, disminuyendo el calor y ajustandose a lo que la literatura plantea. La impermeabilización por su parte, actúa aumentando los grados de calor, tal como lo plantean los autores considerados. Existen factores que sólo resultan siginificativos para la ciudad de Valparaíso, como son la rugosidad de las edificaciones y la exposición de laderas. Ambos fueron evaluados también para la ciudad de Santiago, sin embargo, no se encontraron relaciones.

El reconocimiento de que existen factores ambientales y urbanos que están influenciando en dicho comportamiento, constituye un antecedente valioso de considerar en la gestión ambiental de la ciudad, que permitan definir acuerdos y construir políticas que propendan al mejoramiento de la calidad de vida de sus habitantes. Lo anterior requiere de la incorporación del concepto de red de monitoreo, que sirva a la articulación de las diferentes fuentes de datos meteorológicos actualmente disponibles para las distintas ciudades del país. Si bien es cierto, que se cuenta con datos de 13 estaciones en Valparaíso y 30 estaciones meteorológicas en Santiago, el que pertenezcan a instituciones distintas,con objetivos diversos, constituye una importante limitación, ya que aumenta el nivel de incertidumbre respecto a la utilización de las series de datos de manera conjunta, aun más considerando que no existe información respecto al estado de los instrumentos y la calidad de los datos registrados.

La complejidad derelaciones que determinan la variabilidad de las temperaturas, sugiere que en el futuro, la representación continua 
de las mismas, considere metodologías que faciliten la inclusión y análisis de diversas variables. En tal sentido, métodos de inferencia que puedan incorporarlas, como la regresión múltiple, aparecen como herramientas más adecuadas frente a interpoladores usualmente utilizados como el kriging. Esto requiere ineludiblemente, de la implementación de nuevas estaciones meteorológicas, que logren representar las variables que los modelos destacan, dando especial importancia a la orografía, a la consideración de la escala y a variables que no ha sido posible incorporar en ésta investigación como son, la dirección e intensidad de los flujos de viento.

Los esfuerzos dirigidos a destinar recursos para el registro de nuevos datos, podrían ser muy provechosos para proyectar escenarios futuros de variabilidad climática, asociada a un cambio climático a una mayor escala, no solo en Santiago y Valparaíso, sino que también en otras áreas urbanas, ya que ambas ciudades, son representativas de muchos otros escenarios de vulnerabilidad urbana, y su localización en un área de transición climática, las convierte en un buen referente de la evolución del clima, si es que de ellas se tienen antecedentes.

Para una planificación urbana, que incorpore el clima local, se sugiere poner especial atención al crecimiento futuro estimado de las ciudades, atendiendo a las variables descritas y a la consideración de áreas propensas a concentrar temperaturas extremas. El tratamiento estratégico de variables urbanas y geográficas del paisaje y el reconocimiento de áreas a resguardar y gestionar, es el modo de aprovechar las ventajas comparativas que ofrece el marco en el cual se sitúan ambas ciudades. En tal sentido, la geografía puede integrar conocimiento, y en esa línea contribuir a evaluar y a implementar metodologías que favorezcan el análisis integrado de las problemáticas socio-ambientales, para que sean consideradas en la gestión y planificación urbana, dada su significancia.

\section{REFERENCIAS}

AKBARI, H; POMERANTZ, M; TAHA, H. 2001. Cool surfaces and shade trees to reduce energy improve air quality in urban areas. Solar Energy, 70 (3): 295-310.

ARNFIELD, J. 2003. Two decades of urban climate research: A review of turbulence, exchanges of energy and water, and the urban heat island. International Journal of Climatology, 23: 1 - 26.

ARNOLD, C. \& J. GIBBONS. 1996. Impervious surface coverage: The emergence of a key environmental indicator. Journal of the American Planning Association 62 (2): 243-258.

BOLUND, P. \& S. HUNHAMMAR, 1999. Ecosystem services in urban areas. Ecological Economics, 29: 293 - 301.

CARRERAS, C; MARIN, M; VIDE, J; SABI, J. 1990. Modificaciones térmicas en las ciudades, avances sobre la isla de calor en Barcelona. Documentos de Análisis Geográfico, 17: 51 - 77.

CHAO, R; YAN - YUN, E; LUTZ, K. 2010. Urban climate maps studies: A review. International Journal of Climatology, 31: $2213-2233$

CHIESURA, A. 2004. The role of urban parks for the sustainable city. Landscape and Urban Planning, 68: 129 - 138.

COUTTS, A; BERINGER, J; JIMMI, S; TAPPER, N. 2007. The urban heat island in Melbourne: drivers, spatial and temporal variability, and the vital role of storm water. Monash University. In: www.arts.monash. edu.au/ ges/staff/jberinger/pubs/stormwater 2009.pdf. 
CUADRAT, J., M. SAZ, S. VICENTESERRANO, 2003. Surface wind direction influence on spatial patterns of urban heat island in Zaragoza (Spain).Geophysical Research Abstracts, 5: 02592. European Geophysical Society.

CUADRAT J., VICENTE- SERRANO S., SAZ M. 2005. Los efectos de la urbanización en el clima de Zaragoza (España): La isla de calor y sus factores condicionantes. Boletín de la Asociación de Geógrafos de España, 40: 311-327.

DIRECCIÓN METEOROLÓGICA DE CHILE (DMC). 2001. Climatología Regional. 47 pp. http://164.77.222.61/ climatologia/.

ELIASSON I., 1999.The use of climate knowledge in urban planning. Elsevier, Landscape and urban planning, 48: $31-44$.

ESCOBEDO, F; WAGNER, J; NOWAK, D; LUZ DE LAMAZA, C; RODRIGUEZ, M; CRANE, D. 2008. Analysing the cost effectiveness Santiago Chile's policy using urban forest to improve air quality. Journal of Environmental Management, 86: 148 157.

GEORGAKIS，C; SANTAMOURIS, M; KAISARLIS, G. 2010. The vertical stratification of air temperature in the center of Athens. Journal of Applied Meteorology and Climatology, 49: 1219 - 1232.

GUIJARRO， J. 1998.Influencia de la urbanización en las series termométricas de Baleares. In: IV Reunión de Climatología: El clima y el factor urbano. Madrid. Pp. 305 -314 .

GRIMMOND, S; OKE, T. 1998. Heat storage in urban areas: Local scale observation and evaluation of a simple model. Journal of Applied Meteorology, 38: $922-940$
GRIMMOND, S. 2007. Urbanization and global environmental change: Local effects of urban warming. The Geographical Journal, 173: $83-88$.

HONJO T, NARITA K-I, SUGAWARA H, MIKAMI T, KIMURA K \& KUWATA $\mathbf{N}$, 2003. Observation of cool island effect in urban park (Shinjuku Gyoen). In: $15^{\mathrm{a}}$ Conferencia Internacional de Clima Urbano, Polonia.

INSTITUTO NACIONAL DE ESTADÍSTICAS, INE. 2008. Población y Sociedad: Aspectos Demográficos. Publicación especial disponible en: http:// www.ine.cl/canales/chile_estadistico/ demografia_y_vitales/demografia/pdf/ poblacion_sociedad_enero09.pdf

INTERGOVERNMENTAL PANEL ON CLIMATE CHANGE, IPCC. 1990. Climate Change: The IPCC Scientific Assessment. In. Report prepared for Intergovernmental Panel on Climate Change by Working Group I. (Eds) J.T. Houghton, G.J. Jenkinns \& J.J. Ephraums. Cambridge University Press, Cambridge. 410pp.

INTERGOVERNMENTAL PANEL ON CLIMATE CHANGE, IPCC. 1995. The regional impact of Climate Changes. An assessment of vulnerability. IPCC WMO, UNEP, Cambridge University Press. Cambridge. 1pp

JO, M., K. LEE, B. JUN, B. KWON and Y. JO. 2001. The spatial topographic of urban surface temperature using remotely sensed data and GIS. In: 22nd Asian conference on remote sensing. Singapore. 5 pp.

KUSAKA, H. 2008. Recent progress in urban climate study in Japan. Geographical Review of Japan, 81 (5): 361 - 374.

LAMPTEY, B. 2009. An analytical framework for estimating the urban effect on climate. International Journal of Climatology, 30:72 - 88 . 
LINDBERG, F; ELIASSON, I; HOLMER, B. 2003. Urban geometry and temperature variations.In: Fifth International Conference of Urban Climate, Lodz, Poland. 4 pp.

MENDONCA, F. 2009. Urban heat and urban cold islands: Influences of vegetation and soil surface in some cities, southern Brazil. In: The seventh international Conference of urban Climate. Yokohama, Japan.

MOLINA, M. 2007.Efectos de los tipos de urbanización asociados al crecimiento urbano del área Metropolitana del Gran Santiago sobre la generación y comportamiento de micro islas de calor. Memoria para optar al Título de Geógrafo. Universidad de Chile. Santiago, 118 pp. Inédito

MONTÁVEZ, J; RODRÍGUEZ， A; JIMENEZ, J. 2000.A study of the urban heat island of Granada. International Journal of Climatology, 20: 899 - 911.

NOWAK, D; McPHERSON, E. 1993. Cuantificación del impacto ambiental de los árboles en Chicago. Depósito de documentos de la FAO. Revista Unasylva - No. 173 La silvicultura urbana y periurbana. http:// www.fao.org/docrep/u9300s/u9300s00.htm

OKE, T. 1987. The thermal regime of urban parks in two cities with different summer climates, International Journal of Remote Sensing, 19 (11):2085 - 2104.

PAPARELLI, A; KURBAN, A; CONSULO, M. 2011. Isla de calor y ocupación espacial urbana en San Juan: Análisis evolutivo. Cuadernos de Vivienda y Urbanismo, 4(7): 110 - 120.
PIGEON, G; LEMONSU, A; GRIMMOND, S; DURAND, P; THOURON, O; MASSON, V. 2007. Divergence of turbulence fluxes in the surface layer: The case of a coastal city. Boundary Layer Meteorology, 124: 269 290.

PRASHAD, L, 2004. Urban Materials and Temperature in Phoenix: Connecting Land Use, Socioeconomics, and Vegetation and Relating Ground and Air Variables. M.S. Thesis Defense, Arizona State University. Inédito.

ROMERO, H; MOLINA, M; VÁSQUEZ, A; SMITH, P. 2008. El clima urbano del puerto de Valparaíso: Construcción social del espacio en ciudades costeras. Revista da Facultade de Letras. Universidade do Porto, $2: 103-122$.

RUIZ - FLAÑO, P; ROMERO, L; MÁYER, P; HERNÁNDEZ, A. 2008. La isla de calor en Las Palmas de Gran Canaria: Intensidad, distribución y factores condicionantes. Boletín de la Asociación de Geógrafos de España, 47: 157 - 173.

SALAMANCA, F. 2010. Desarrollo de modelos numéricos para investigar la isla de calor en ciudades y estudio de la sensibilidad de distintos parámetros urbanos. Facultad de Ciencias Físicas. Universidad Complutense de Madrid. Tesis para optar al grado de Doctor. Madrid, 212 pp. Inédito.

SARRICOLEA, P. 2008. Análisis de la sustentabilidad del crecimiento urbano de la ciudad de Santiago y sus efectos sobre la configuración de las temperaturas superficiales. Tesis para optar al grado de Magíster. Universidad de Chile. Santiago. 122 pp. Inédito. 
SARRICOLEA, P; ALISTE, E; CASTRO, P; ESCOBEDO, C. 2008. Análisis de la máxima intensidad de la isla de calor urbana nocturna de la ciudad de Rancagua (Chile) y sus factores explicativos. Revista de Climatología, 8: $71-84$.

SARRICOLEA, P; ROMERO, H. 2009. Análisis de los factores condicionantes sobre las temperaturas de emisión superficial en el Área Metropolitana de Valparaíso. Revista Arquitectura, Ciudad y Entorno, 14: 79 - 96.

SARRICOLEA, P. 2012. La isla de calor urbana de superficie y sus factores condicionantes: El caso del área metropolitana de Santiago. Memoria para optar al grado de Doctor. Universitat de Barcelona. Barcelona. 332 pp. Inédito.

SPROKEN - SMITH, R; OKE, T. 1999. Scale modeling of nocturnal cooling in urban parks. Boundary Layer Meteorology. 93: $287-312$.

STEWART, I.D. AND OKE, T. 2009. Classifying urban climate field sites by "local climate zones": The case of Nagano, Japan. In: Preprint, Seventh International Conference on Urban Climate, Yokohama, Japan.

VÁSQUEZ, A; ROMERO, H. 2007. El libre mercado de las áreas urbanas y la falta de justicia ambiental en la disponibilidad de áreas verdes en Santiago de Chile. In: IX Coloquio Internacional de Geocrítica. Porto Alegre. Brasil.

VERÓN, E. 2010. Estimación de la isla de calor en Santa Teresita, partido de la costa, provincia de Buenos Aires, Argentina. Revista Geográfica de América Central, 45: $129-148$.

VILELA, J. 2004.Distribución del arbolado urbano en la ciudad de Fuenlabrada y su contribución a la calidad del aire. Revista Ciudad y Territorio, 36: $419-427$.
WIENERT, U; KUTTLER, W. 2005. A dependence of the urban heat island intensity on latitude: A statistical approach. Meteorologische Zeitschrift, 14 (5): 677 686.

YUAN, F; BAUER, M. 2007.Comparison off impervious surface area and normalized difference vegetation index as indicators of surface urban heat island effects in Landsat Imagery. Remote Sensing of Environment, 106: $375-386$.

ZHANG, Z; JI, M; SHU, J; DENG, Z; WU, Y. 2008. Surface urban heat island in Shanghai, China: Examing the relationship between land surface temperature and impervious surface fraction derived from Landsat ETM+ Imagery. The international Achieves of Photogrammetric, Remote Sensing and Spatial Information Sciences, 37: $601-606$. 